\title{
CRUCIAL ROLE OF INELASTIC BACKGROUND SUBTRACTION IN IDENTIFYING NON-FERMI LIQUID BEHAVIOR IN EXISTING ARPES LINESHAPE DATA*
}

\author{
L.Z. LIU, R.O. ANDERSON and J.W. ALLEN \\ Randall Laboratory of Physics, The University of Michigan, Ann Arbor, MI 48109-1120

\begin{abstract}
We have fit angle-resolved photoemission spectra of $\mathrm{Bi}_{2} \mathrm{Sr}_{2} \mathrm{BaCu}_{2} \mathrm{O}_{8}$ taken by Olson et al. ${ }^{1}$ using normal and marginal Fermi liquid self energies. Both theories fit the data equally well provided a sufficiently large inelastic background is included. However, the required backgrounds are, respectively, 60 and 15 times larger than that given by usual background subtraction procedures.

*work supported by NSF grant No. DMR-87-21654 and DoE grant No. DE-FG0290ER45416.
\end{abstract}

Keywords: superconductor,' photoemission, Fermi liquid, Marginal Fermi liquid, self energy

\section{Introduction}

Angle-resolved photoemission spectroscopy (ARPES) has had a major impact on the thinking about high temperature superconductors by providing the first evidence for the existence of a Fermi surface having a volume consistent with Luttinger's counting theorem. ${ }^{1,2}$ Beyond this, the ARPES lineshape has also attracted much interest. Olson et al. have analyzed the lineshapes in their data for $\mathrm{Bi}_{2} \mathrm{Sr}_{2} \mathrm{BaCu}_{2} \mathrm{O}_{8}$ along the $\Gamma$-X direction by an ansatz using Lorentzians with widths dependent on peak binding energy $E_{B}$, and reported that better fits could be achieved with widths $\propto E_{B}$ than with widths $\propto E_{B}^{2}$. This has been taken as evidence supporting marginal Fermi liquid behavior in the normal state. The asymmetry of the lineshape has also been thought to show marginal ${ }^{3}$ or Luttinger liquid ${ }^{4}$ behavior, although the asymmetry has been fit in the ansatz of Olson et al. by taking account of the nonzero angular aperture of the experiment. The ansatz of Olson et al. corresponds neither to Fermi liquid nor marginal Fermi liquid behavior, as we point out below. We present here the result of fits of the data of Olson et al. using both Fermi liquid and marginal Fermi liquid lineshapes, with and without the effect of the nonzero angular aperture.

\section{Lineshape analysis procedure}

The ARPES lineshape is given by the spectral weight of the single particle Green's function $G$, assuming, as usual, the validity of the sudden approximation in describing the photoexcitation event. The expression for the Green's function is $G(\mathbf{k}, \omega)^{-1}=\omega-\epsilon_{\mathbf{k}}-\Sigma(\mathbf{k}, \omega)$, where $\epsilon_{\mathbf{k}}$ is the free-particle excitation energy and $\Sigma$ is the self energy. So the spectral weight is given by

$$
A(\mathbf{k}, w)=\frac{1}{\pi} \frac{\operatorname{Im} \Sigma(\mathbf{k}, w)}{\left[w-\epsilon_{\mathbf{k}}-\operatorname{Re} \Sigma(\mathbf{k}, w)\right]^{2}+[\operatorname{Im} \Sigma(\mathbf{k}, w)]^{2}}
$$

Fermi liquid (FL) and marginal Fermi liquid (MFL) theories have different self energies. The self energy for Fermi liquid theory, assumed here to be momentum independent, is taken to be

$$
\Sigma(\mathbf{k}, \omega)=\alpha \omega+i \beta \omega^{2} .
$$

In constructing the spectral function, we define new quantities $\epsilon_{\mathbf{k}}^{\prime}=\frac{\epsilon_{k}}{1-\alpha}, \beta^{\prime}=\frac{\beta}{1-\alpha}$, and make an overall scaling of $A(k, \omega)$ by $1-\alpha$. The self energy for MFL theory is ${ }^{3}$

$$
\Sigma(\mathbf{k}, \omega) \sim g^{2} N(0)^{2}\left(\omega \ln \frac{x}{\omega_{c}}-i \frac{\pi}{2} x\right)
$$

where $x=\max (|\omega|, T), \omega_{c}$ is an ultraviolet cutoff, and $g^{2} N(0)^{2}$ is defined as $g^{\prime}$. The general appearance of these lineshapes for $|\mathbf{k}|$ less than the Fermi momentum $k_{F}$ is shown in Fig. 1. 
Neither lineshape has emission at the Fermi energy $E_{F}$ (except when $|\mathbf{k}|=k_{F}$ ), in contrast to the ansatz of Olson et al., in which, for every $\mathbf{k}$, there is a Lorentzian cut off at $E_{F}$ by the Fermi distribution function. It is important for the fits we present below that even for $|\mathbf{k}|>k_{F}$ there is spectral weight below $E_{F}$, just as there is weight above $E_{F}$ in the curves of Fig. 1 .

To fit the data to FL theory, $\beta^{\prime}$ is fixed and then the bare $\epsilon_{\mathbf{k}}^{\prime}$ is varied for each spectrum to get a reasonable peak position. This spectrum is convolved with a Gaussian to approximate the instrument resolution of $34 \mathrm{meV}$. Finally, a standard ${ }^{5}$ inelastic background is added to every point $\mathrm{E}$ that is a fraction of the area under the theoretical curve between $\mathrm{E}$ and the Fermi level.

$$
A(E)=A_{\text {theor }}(E)+\kappa \int_{E}^{E_{F}} A_{\text {theor }}(x) d x
$$

The amount of background added $\kappa$ is fixed for all spectra. By fixing $\kappa$ and $\beta^{\prime}$ and varying the one parameter $\epsilon_{k}^{\prime}$ between different spectra, reasonable fits are eventually found for the Fermi liquid theory as shown in Fig. 2a. The background in each spectrum is also shown in the figure. To include the $2^{\circ}$ finite angular resolution (equivalent to a momentum uncertainty $\Delta \mathbf{k})$, the dispersion is found from the $\epsilon_{\mathbf{k}}^{\prime}$ fit and a series of normalized spectral functions are summed together assuming a square angular window. The fits as shown in Fig. 3a are not drastically altered by the addition of the finite angular acceptance, except that for $|\mathbf{k}|$ very near $k_{F}$, the window includes contribution from both sides of the Fermi surface, which have different $\omega$ dependences (see Fig. 1), and thus produce a double peak shape that is not seen in the data. In addition, the spectral weight becomes more asymmetric, as found by Olson et al, and $\epsilon_{\mathbf{k}}^{\prime}$ changes somewhat.

The fitting for MFL theory is similar, but now the fixed parameters for all spectra are $\omega_{c}$, $g^{\prime}$, and background $\kappa$. Only $\epsilon_{\mathbf{k}}$ is varied between different spectra. The MFL fits as well as the parameter values used are shown in Figs. $2 \mathrm{~b}$ and $3 \mathrm{~b}$. The double peak shapes pointed out above for the FL do not occur for the MFL.

\section{Discussion of Results}

The fits obtained with either lineshape are comparably good apart from the FL fit with the double peak shape which is not seen in experiment. There is some emission at $E_{F}$ that is not well fit by either theory. The quadratic curvature of the FL theory near $E_{F}$ misses much of this feature, while the MFL theory's linear dependence tends to improve the appearance of the fit. However, even in the MFL case, the inflection point between the $E_{F}$ emission and the emission at deeper binding energy is not fit. An important difference between our fits and those of Olson et al. is that for $|\mathbf{k}|>k_{F}$ (the uppermost curves in the data set) we assign a large portion of the emission as real spectral weight rather than assuming all of it is inelastic background. We also note that the Lorentzian ansatz of Olson et al. gives fits which are, fortuitously in our opinion, better for the $E_{F}$ feature.

The main observable difference in the two fits is in the falloff of the tail at higher energies, which then requires quite different assumptions about the magnitude of the inelastic background. Although the self-energy approximations used are strictly valid for energies near $E_{F}$, the tendency of the MFL to have more incoherent spectral weight displaced to higher energy than for the FL is physically significant. For the MFL lineshape the cutoff energy used ${ }^{3}$ was $_{c}=0.15 \mathrm{eV}$, and the spectral function should be reliable to perhaps $2 \omega_{c}$ or $0.3 \mathrm{eV}$.

The crucial point is that for both theories the amount of inelastic background is much larger than the background one obtains from the normal procedure of simply applying our background algorithm stated above, to the entire valence band. This is seen in Fig. 4 which shows an ARPES spectrum for $\mathrm{Bi}_{2} \mathrm{Sr}_{2} \mathrm{BaCu}_{2} \mathrm{O}_{8}$ taken at the same photon energy $22 \mathrm{eV}$, as the data we are fitting. The background in the first $0.5 \mathrm{eV}$ below $E_{F}$ is negligible. Quantitatively, the background coefficients needed for the FL and MFL fits are, respectively, 60 and 15 times larger than that for the background of Fig. 4. We conclude that neither lineshape falls off slowly enough, assuming that there is no quantitatively important contribution in this binding energy range from the unexplained $\mathrm{E}_{F}$ emission discussed above. 

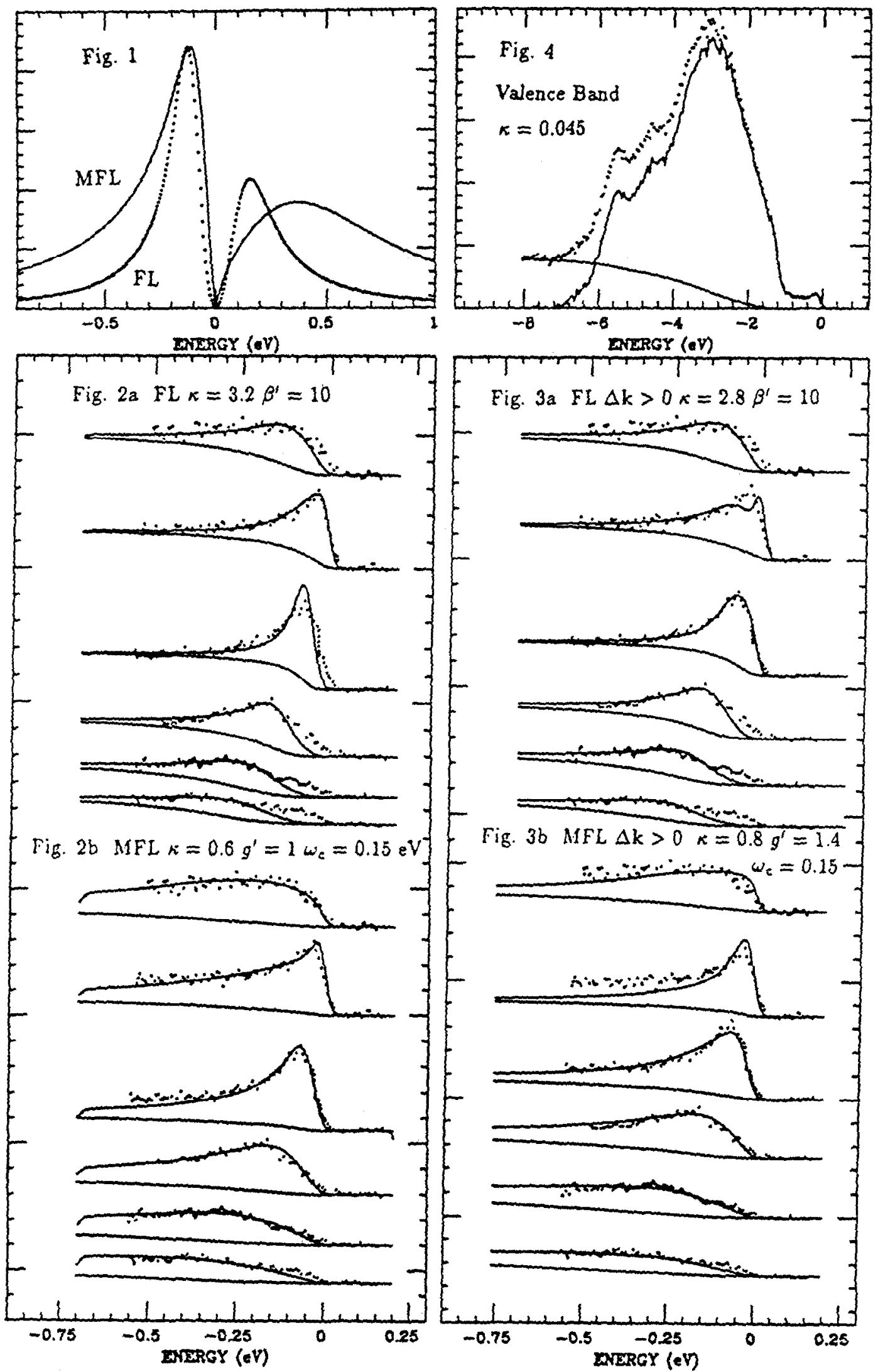


\section{Summary}

If the background $\kappa$ is adjusted, the FL and MFL fits are equally good. The finite angular resolution included for realism does not affect this conclusion. If we use an inelastic background set by the entire valence band, which we believe to be appropriate, and if we assume that the unexplained $\mathrm{E}_{F}$ feature does not extend far from $\mathrm{E}_{F}$, then neither the FL nor MFL theories can fit the slow falloff of the spectrum at higher energies, although the MFL lineshape is certainly better.

\section{References}

${ }^{1}$ Olson, C.G. et al., Phys. Rev. B. 42, 381 (1990).

${ }^{2}$ Manzke, R. et al., Physica Scripta 41, 579 (1990).

${ }^{3}$ Varma, C.M. et al., Phys. Rev. Lett. 63, 1996 (1989).

${ }^{4}$ Anderson, P.W., Phys. Rev. Lett. 64, 1839 (1990).

${ }^{5}$ Allen, J.W. et al., Adv. in Phys. 35, 275 (1986). 